\title{
Understanding the People Who Provide Care
}

$\mathrm{P}$ OPLE ARE CENTRAL TO HEALTHCARE - THOSE WHO ARE CARED FOR AND THOSE who care for others. According to Statistics Canada, more than 1.6 million Canadians worked in health and social assistance in 2010 (Statistics Canada 2011). Even more - over 2 million - are informal caregivers (Statistics Canada 2002). These family, friends and neighbours provide enormous amounts of care and support across the country.

This edition of Healthcare Policy/Politiques de Santé focuses on health human resources, including issues relevant to both formal and informal caregivers. This is one of the most complex and enduring areas of health policy and of health services and policy research. While the issues are sometimes reduced to simple "do we need more or fewer?" debates, they go far beyond such a simple analysis, as this edition of the journal demonstrates. Think of the impact of shifting scopes of practice, such as recent expansions in the healthcare professions that can prescribe medications; technology changes that have the potential to fundamentally alter who delivers care and how, such as new telestroke programs or remote monitoring services in the home; or clinicians working together in new ways, as in the recently announced emergency centres with paramedics in Nova Scotia (Government of Nova Scotia 2011).

In this issue of the journal, authors provide new evidence and new perspectives on thorny health human resources questions, from entry to practice to retirement. Bob Evans starts us off with a commentary on the potential implications of changing physician supply. Brenda Gamble and colleagues also look at supply questions, but with a focus on respiratory therapy and whether pushes to shift care to community-based or home settings have met with success.

How to influence health human resources policy is also a focus of the paper by Vanessa Burkoski, Joshua Tepper and Sue Matthews. They look at collaborative engagement in the development of Ontario's Nursing Graduate Guarantee, an effort to support full-time employment for new graduate nurses. This policy area also caught the interest of Andrea Baumann, Mabel Hunsberger and Mary Crea-Arsenio. With others in Canada and abroad contemplating similar efforts or tinkering with existing programs, these papers are timely. Facilitating learning from others' experiences is also the hope of Mylaine Breton and colleagues, who ask whether there are lessons from Ontario's family health teams (FHTs) for those interested in Quebec's family medicine group (FMG) model. Similarly, Joanne Kim and her co-authors introduce a potential triage program as a way to reduce the costs of diagnostic imaging for certain spinal disorders.

Rounding out the issue are four papers that cast a broad net in terms of the stakeholders involved in different aspects of health policy. The first, by Sioban Nelson and colleagues, examines the implications of recent changes in immigration policy in Canada for health 


\section{Editorial}

human resources. In the second, Gayle Restall and Joseph Kaufert explore how context shapes citizen-user involvement in policy making, with a specific focus on mental health and housing services. Mental health is also the area on which Elliot Goldner and colleagues focus their knowledge translation scoping review. Last, but certainly not least, is Meredith Lilly's discussion and debate paper on the role of employers in creating workplaces that support employees who are caregivers for family and friends.

In an issue with several papers focused on mental health policy and practice, it seems particularly appropriate to welcome Eric Latimer to the Healthcare Policy/Politiques de Santé editorial team. Eric has appointments in the departments of Psychiatry and Epidemiology, Biostatistics and Occupational Health at McGill University. He is also a researcher at the Douglas Institute and brings a wide range of expertise and experience to complement that of our other editors. Please join me in welcoming him to the team.

JENNIFER ZELMER, BSC, MA, PHD

Editor-in-chief

\section{REFERENCES}

Government of Nova Scotia. 2011 (April 6). "Nova Scotia's First Collaborative Emergency Centre Announced." News release. Retrieved October 24, 2011. <http://gov.ns.ca/news/smr/2011-04-06-CEC/>.

Statistics Canada. 2002. General Social Survey. Cycle 16: Aging and Social Support. Ottawa: Author. Retrieved October 24, 2011. <http://www.statcan.gc.ca/pub/89-583-x/index-eng.htm>.

Statistics Canada. 2011 (March 31). "Employment, Health Care and Social Assistance, by Province and Territory, 2006-2010." CANSIM table 281-0024 and Catalogue no. 72-002-XIB. Retrieved October 24, 2011. <http:// www40.statcan.ca/101/cst01/HEALTH22-eng.htm>. 


\section{Comprendre les personnes qui fournissent des soins}

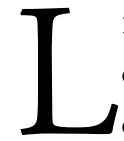

ES GeNS SONT AU CENTRE DES SoINS DE SANTÉ - TANT CEUX QUi LES ReÇOIVENT que ceux qui les donnent. Selon Statistique Canada, plus de 1,6 million de Canadiens et Canadiennes travaillaient dans le secteur des soins de santé et de l'assistance sociale en 2010 (Statistique Canada, 2011). Bien plus de personnes encore, soit plus de deux millions, fournissent des soins de façon officieuse (Statistique Canada, 2002). Ces parents, amis et voisins offrent d'énormes quantités de soins et beaucoup de soutien dans l'ensemble du pays.

Ce numéro de Politiques de Santé/Healthcare Policy porte sur les ressources humaines en santé, notamment les enjeux qui touchent aux fournisseurs de soins, qu'ils soient officiels ou officieux. En termes de politique, de recherche et de services de santé, il sagit de l'un des secteurs les plus complexes et récurrents. Bien que la question soit souvent réduite à de simples débats sur la nécessité de recourir davantage ou non à de tels types de soins, l'enjeu est bien plus complexe, comme le démontrent les articles de ce numéro. Pensons à l'impact des changements dans le champs d'activité, tels que la récente expansion de professionnels de la santé qui peuvent prescrire des médicaments; pensons aux changements technologiques qui transforment la nature et la portée des prestataires de soins, tels que les nouveaux programmes de télé-AVC ou les services de télésurveillance à domicile; ou pensons aux cliniciens qui collaborent de façons novatrices, tels que les centres de premiers secours dotés de techniciens médicaux d'urgence annoncés récemment en Nouvelle-Écosse (Gouvernement de la Nouvelle-Écosse, 2011).

Dans ce numéro, les auteurs présentent de nouvelles données et points de vue sur d'épineuses questions liées aux ressources humaines en santé, de l'entrée dans la pratique à la retraite. Bob Evans commente les répercussions potentielles des changements dans l'apport de médecins sur le marché du travail. Brenda Gamble et ses collaborateurs se penchent également sur la question de l'apport en termes de ressources humaines, mais du point de vue de l'inhalothérapie, notamment pour savoir si les incitatifs pour transférer les points de service vers les secteurs communautaire et domiciliaire ont porté fruit.

Larticle de Vanessa Burkoski, Joshua Tepper et Sue Matthews porte également sur la façon d'influencer les politiques sur les ressources humaines en santé. Ils se penchent sur les collaborations dans la création de la Garantie d'emploi pour les diplômés en soins infirmiers en Ontario, une initiative qui vise à appuyer l'embauche à temps plein des nouveaux diplômés en soins infirmiers. Cette politique a également attiré l'attention d'Andrea Baumann, de Mabel Hunsberger et de Mary Crea-Arsenio. Ces articles ont un caractère très opportun alors quau Canada et à l'étranger d'autres chercheurs et intervenants étudient des initiatives similaires ou sont aux prises avec des programmes déjà en place. Mylaine Breton et ses collaborateurs ten- 
tent également de favoriser l'apprentissage à l'aide des expériences des autres, notamment en repérant les leçons que le modèle ontarien des équipes de santé familiale peut offrir au modèle des groupes de médecine de famille au Québec. Semblablement, Joanne Kim et ses collaborateurs présentent un programme hypothétique de triage comme solution pour réduire les coûts d'imagerie diagnostique dans certains cas de problèmes de la colonne vertébrale.

En complément de numéro, quatre articles font appel à une vaste gamme d'intervenants impliqués dans divers aspects des politiques de santé. Le premier, de Sioban Nelson et collaborateurs, examine les répercussions de récents changements dans les politiques d'immigration au Canada sur les ressources humaines en santé. Le deuxième, de Gayle Restall et Joseph Kaufert, examine comment le contexte influe sur l'implication des citoyens dans l'élaboration de politiques, avec un accent particulier sur la santé mentale et les services à domicile. La santé mentale est également le sujet de l'analyse sur l'échange de connaissances effectuée par Elliot Goldner et ses collaborateurs. Pour terminer, l'article de discussion et de débat de Meredith Lilly porte sur le rôle des employeurs dans la création de milieux de travail qui appuient les employés agissant comme aidants naturels auprès d'un parent ou d'un ami.

Ce numéro, qui comporte de nombreux articles sur les politiques et la pratique en santé mentale, est l'occasion idéale de souhaiter la bienvenue à Eric Latimer dans l'équipe de rédaction de Politiques de Santé/Healthcare Policy. M. Latimer occupe des postes au Département de psychiatrie et au Département d'épidémiologie, biostatistique et santé au travail de l'Université McGill. Il est également chercheur à l'Institut Douglas et apportera une vaste expérience complémentaire à notre équipe de rédaction. Je vous invite à lui souhaiter la bienvenue au sein de l'équipe.

JENNIFER ZELMER, BSC, MA, PHD

Rédactrice en chef

\section{RÉFÉRENCES}

Gouvernement de la Nouvelle-Écosse. 2011 (6 avril). “Nova Scotia’s First Collaborative Emergency Centre Announced." Communiqué de presse. Consulté le 24 octobre 2011. <http://gov.ns.ca/news/smr/2011-04-06$\mathrm{CEC} />$.

Statistique Canada. 2002. L’enquête sociale générale de 2002, cycle 16 : Vieillissement et soutien social. Ottawa : auteur. Consulté le 24 octobre 2011. <http://www.statcan.gc.ca/pub/89-583-x/index-fra.htm>.

Statistique Canada. 2011 (mars 31). «Emploi, soins de santé et assistance sociale, par province et territoire, 20062010. » Tableau CANSIM 281-0024 et produit no. 72-002-XIB au catalogue. Consulté le 24 octobre 2011. <http://www40.statcan.ca/102/cst01/health22-fra.htm>. 